\title{
Estimación numérica del hidrograma de salida de una presa debido a su ruptura
}

\section{Numerical Approach to an Outflow Hydrograph of a Dambreak}

\author{
Fuentes-Mariles O.A. \\ Instituto de Ingeniería, Facultad de Ingeniería \\ Universidad Nacional Autónoma de México \\ E-mail:ofm@pumas.ingen.unam.mx \\ Arganis-Juárez M.L. \\ Instituto de Ingeniería, Facultad de Ingeniería \\ Universidad Nacional Autónoma de México \\ E-mail:MarganisJ@iingen.unam.mx \\ De Luna-Cruz F. \\ Instituto de Ingeniería \\ Universidad Nacional Autónoma de México \\ E-mail:fluc@pumas..ingen.unam.mx \\ Franco V. \\ Instituto de Ingeniería, Facultad de Ingeniería \\ Universidad Nacional Autónoma de México \\ E-mail:vfr@pumas.iingen.unam.mx
}

(Recibido: agosto de 2008; reevaluado: julio de 2009; aceptado: febrero de 2010)

\section{Resumen}

En este trabajo se obtuvieron hidrogramas de salida de una presa ante la eventual ruptura de su cortina; para ello, se utilizó un algoritmo numérico para el tránsito de la avenida por el embalse que toma en cuenta los cambios en el ancho de la brecha por donde egresa el agua y en la altura de la cortina de la presa; los hidrogramas de salida fueron obtenidos para tres escenarios distintos; finalmente los resultados fueron utilizados en un modelo del cálculo de la onda de ruptura de presas para obtener el flujo de agua que se presentaría por el cauce ubicado abajo de la cortina para estimar las consecuencias de la descarga de un gran volumen de agua en un lapso de varias horas. El caso de estudio fue el proyecto hidroeléctrico de La Parota, en el estado de Guerrero, México.

Descriptores: Ruptura de presa, ancho de brecha, hidrograma de salida, proyecto hidroeléctrico La Parota, Gro., Méx., tránsito de avenidas, onda de ruptura.

\section{Abstract}

In this paper ouflow hydrographs of a dam breach were obtained by means of a reservoir numerical flood routing algorithm which takes into count the variations in the width of the breach and the height of the dam; the obtained outflow hydrographs were considered for three 
DOI: http://dx.doi.org/10.22201/fi.25940732e.2010.11n3.023

Estimación numérica del hidrograma de salida de una presa debido a su ruptura

\begin{abstract}
different situations; finally these hydrographs were used as input data in a wave propagation model of a river located downstream of the hydropower project La Parota, Gro., Mexico.
\end{abstract}

Keywords: Dam break, breach width, outflow hydrograph, hydropower project La Parota, Gro., Mex., flood routing, flood wave.

\section{Introducción}

Cuando la cortina de una presa se rompe, o bien, un bordo o dique fallan, se libera un gran volumen de agua en muy poco tiempo. El agua que sale bruscamente forma una avenida que se traslada aguas abajo con una fuerte velocidad, por lo que el flujo de agua posee una gran capacidad de transporte de sólidos y desarrolla una fuerza potencial de arrastre sobre objetos que se encuentren a su paso. Esta avenida suele salirse del cauce del río y provocar inundaciones, que pueden ocasionar la pérdida de vidas humanas o graves daños materiales. La determinación de las zonas anegadas, sus profundidades, así como las velocidades de las corrientes en los cauces de los ríos y zonas aledañas a donde llega el agua, permite estimar los efectos que tendrán los flujos de agua sobre la infraestructura urbana, áreas productivas y en la población, a partir de los cuales se podrían establecer medidas y planes de mitigación de daños ante un fenómeno de esta naturaleza.

Un aspecto fundamental en el estudio de los flujos de agua, se refiere a la salida repentina del volumen de agua contenido por la cortina, para lo cual, se requiere determinar los gastos de egreso una vez que comienza a romperse. Existen distintos criterios para estimar el gasto de salida por la brecha que se abre en la cortina cuando está la falla, el cual depende directamente de la forma en que evoluciona en el tiempo; tal es el caso del método del National Weather Service, presentado por Wetmore et al. (1991), como parte del modelo simplificado de pronóstico de la avenida de una ruptura de presa (SMPDBK). Por otra parte, Dodson et al. (1997) emitieron un documento técnico en el que recopilaron varios procedimientos para la estimación del gasto de pico del hidrograma que se forma por la falla, con el fin de hacer un análisis de estos resultados antes de aplicarse a un modelo de tránsito aguas abajo de la presa. También Aureli y Mignosa (2001) hicieron el modelo de laboratorio de un dique para establecer los cambios que sufre la brecha que se forma en la cortina durante su falla y del comportamiento del flujo bidimensional aguas abajo del embalse.

En los estudios de este tipo es fundamental la evaluación de los gastos de los flujos de agua que salen de la presa cuando inicia la falla de su cortina. Para ello, se propuso emplear un esquema numérico de alta resolución que tome en cuenta la fuerte pendiente de la superficie libre de la corriente y los regímenes de flujo en el cauce.

\section{Sitio del caso de estudio}

El Proyecto Hidroeléctrico La Parota se encuentra sobre el río Papagayo, en la porción sur-central del estado de Guerrero, México. Sus coordenadas geográficas son:

$16^{\circ} 56^{\prime} 03^{\prime \prime}$ de Latitud Norte y $99^{\circ} 37^{\prime} 32^{\prime \prime}$ de Longitud Oeste.

La cuenca del río Papagayo se localiza en el estado de Guerrero; es la más importante de la Región Hidrológica de Costa Chica y reúne las aguas de los ríos Omitlán, Azul o Petaquillas y Papagayo; éste último desemboca en el océano Pacífico (figura 1); en dicha cuenca existen tres estaciones hidrométricas, la de Agua Salada, El Puente y La Parota. Sobre el cauce, casi en la unión de los ríos Omitlán y Papagayo, se sitúa la presa La Venta y aguas abajo de ésta se ubicará el proyecto hidroeléctrico La Parota.

El gasto máximo instantáneo estimado en la estación hidrométrica La Parota durante el periodo que ha operado sobre el río Papagayo es de $11,653 \mathrm{~m}^{3} / \mathrm{s}$ y corresponde a una lectura de escala de $16.06 \mathrm{~m}$, la cual fue registrada el 26 de septiembre de 1967 . Este gasto se obtuvo a partir de la extrapolación de una curva elevaciones-gastos, construida con datos de aforos correspondientes a elevaciones de superficie libre menores a la mencionada.

El gasto mínimo es cero, debido a la operación de la presa La Venta, ubicada $35 \mathrm{~km}$ aguas arriba del sitio donde se ubicará La Parota, la cual llega a cortar totalmente el flujo de agua del río Papagayo. La cortina del P.H. La Parota, Gro. será de enrocamiento con núcleo de arcilla y cara de concreto (Fuentes et al., 2005).

\section{Metodología}

Modelo matemático para obtener la avenida que sale del vaso de la presa

Si se considera que la curva elevaciones-capacidades del embalse de una presa (figura 2) está dada por la ecuación 1. 


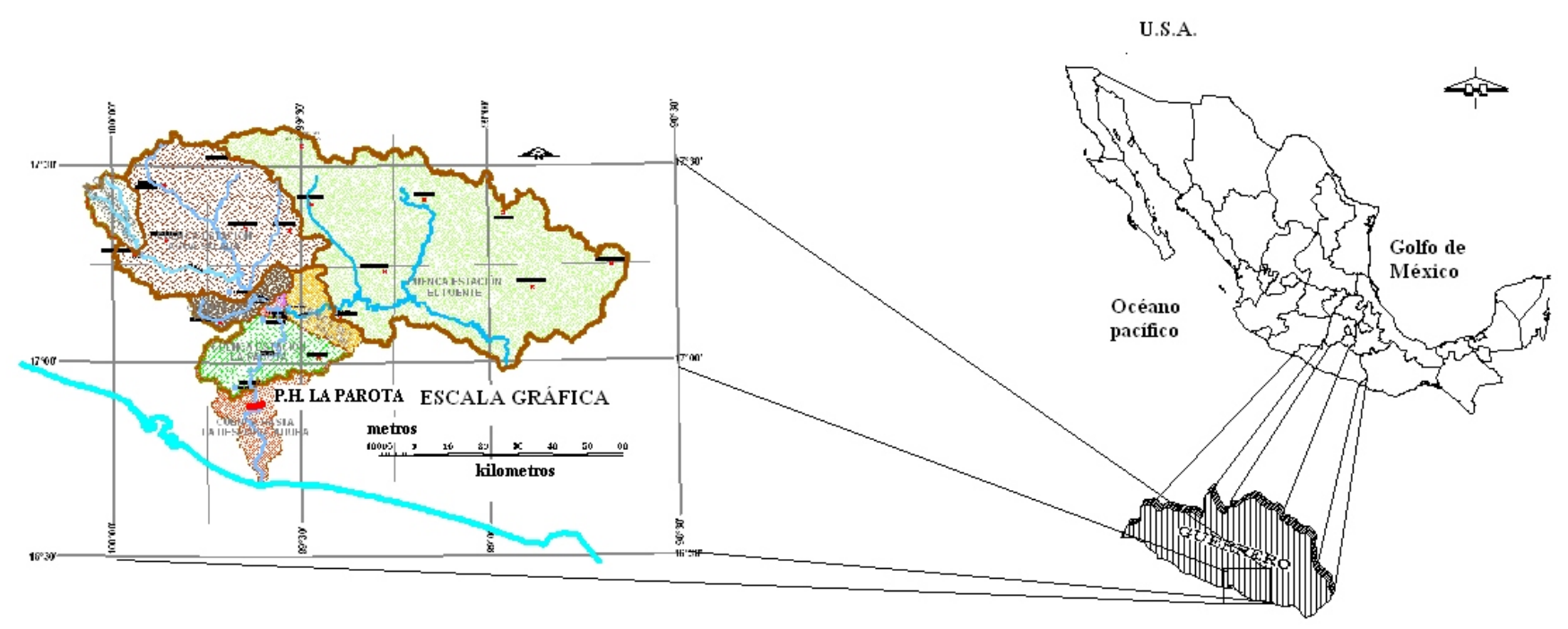

Figura 1. Ubicación de la cuenca del río Papagayo
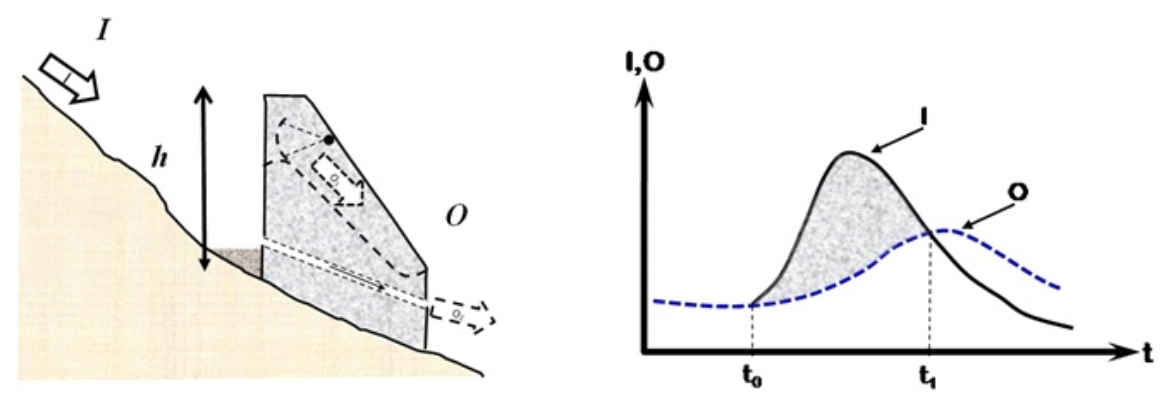

Figura 2. Representación esquemática de los ingresos y egresos en un embalse

$V=K h^{N}$

Donde $\mathrm{V}$ es el volumen almacenado $\left(\mathrm{en}^{3} / \mathrm{s}\right) ; h$ elevación en el embalse (en m), K, N son coeficientes (adimensionales); para el caso del vaso de la futura presa, se encontró que $\mathrm{K}=11.317$ y $\mathrm{N}=3.9101$.

Derivando la ecuación 1 con respecto al tiempo

$$
\frac{d V}{d t}=\mathrm{KN} h^{N-1} \frac{d h}{d t}
$$

Aplicando la ecuación de continuidad al volumen de agua almacenada en la presa (volumen de control), se tiene que el cambio del almacenamiento en el tiempo es igual al gasto de ingreso menos el de salida, es decir,

$$
\mathrm{KN} h^{N-1} \frac{d h}{d t}=I-O
$$

donde $I$ y $O$ son los gastos de entrada y de salida (en $\mathrm{m}^{3} / \mathrm{s}$ ), respectivamente.

La ecuación 3 fue resuelta numéricamente por el método predictor corrector de Euler-Gauss (Chapra y Canale, 1999) con las ecuaciones siguientes:

$$
\begin{aligned}
& \widetilde{h}_{j+1}=h_{j}+\frac{I_{j}-O_{j}}{\mathrm{KN} h_{j}^{N-1}} \Delta t \\
& h_{j+1}=h_{j}+\left[\frac{I_{j}-O_{j}}{h_{j}^{N-1}}+\frac{I_{j+1}-\widetilde{O}_{j+1}}{\widetilde{h}_{j+1}^{N-1}}\right] \frac{\Delta t}{2 K N} .
\end{aligned}
$$

La expresión (4) se le conoce como ecuación de predicción del valor de $h_{j+1}\left(\widetilde{h}_{j+1}\right.$ es la predicción de $\left.h_{j+1}\right)$ y la (5) es la ecuación de corrección de dicho valor $h_{j+1}$. 
Para la estimación del gasto de salida por la abertura que se forma en la cortina, se considera que el agua sale por una sección que se asemeja a un vertedor rectangular, por lo que:

$$
O=\frac{2}{3} \sqrt{2 g \mu b h^{13 / 2}}
$$

donde $g$ es la aceleración de la gravedad $\left(e n \mathrm{~m} / \mathrm{s}^{2}\right) ; b$ es el ancho del vertedor (en $\mathrm{m}$ ), $\mu$ es el coeficiente de descarga (adimensional); $h$ es la elevación del agua en el vaso de la presa (en $\mathrm{m}$ ). Cuando se toma el valor de la aceleración local del sitio igual a $9.81 \mathrm{~m} / \mathrm{s}^{2}$ y del coeficiente $\mu$ como 0.616 se tiene que la ecuación 6 se transforma en:

$$
O=1.819 b h^{13 / 2}
$$

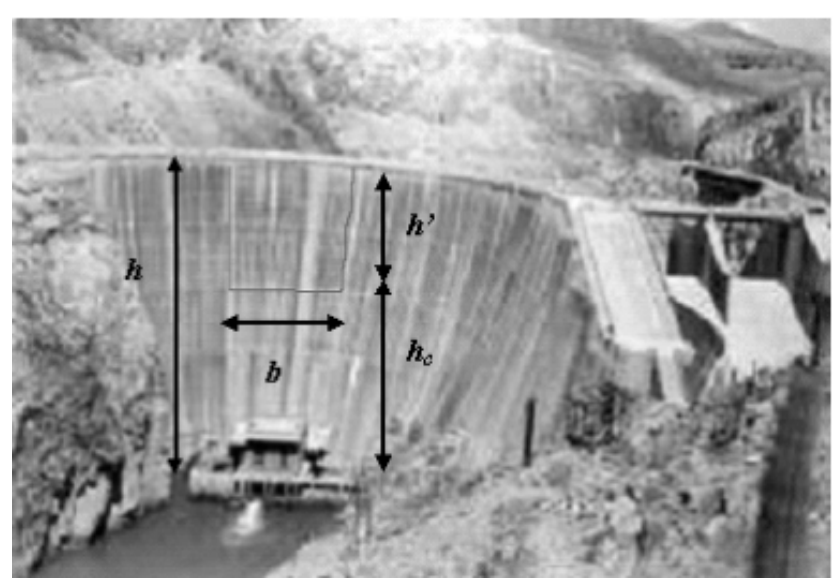

Figura 3. Consideraciones para la carga $h^{\prime}$

donde: $h^{\prime}=h-h_{c}$, siendo $h_{c}$ la elevación (en m) de la supuesta cresta vertedora (figura 3 ).

El gasto de salida está definido por la función siguiente

$$
O=\left\{\begin{array}{cc}
1.819 b(h-h c)^{3 / 2} & h>h_{c} \\
0 & h \leq h_{c}
\end{array}\right.
$$

De acuerdo con el estudio de Fuentes et al. (2005) y a partir de la información recabada relativa a la forma de brechas de presas que han fallado en distintas partes del mundo, formadas por diversos materiales, no sólo de tierra, sino de combinaciones entre materiales tales como enrocamiento y tierra, tierra con cara de concreto; para una presa de material homogéneo (Coleman et al., 2002, Chinnarasri, 2004), se propuso que el ancho de la brecha cambie de acuerdo a una ley parabólica en función del tiempo y que la altura de la cresta $(h)$ lo haga de acuerdo con una función potencial, también en función del tiempo, como se ejemplifica en las figuras 4 y 5 . Adicionalmente, en la figura 6 se muestra un hidrograma de salida por la ruptura de un bordo homogéneo de arena volcánica obtenido en el Laboratorio de Hidráulica (Rivera y Fuentes, 2009).

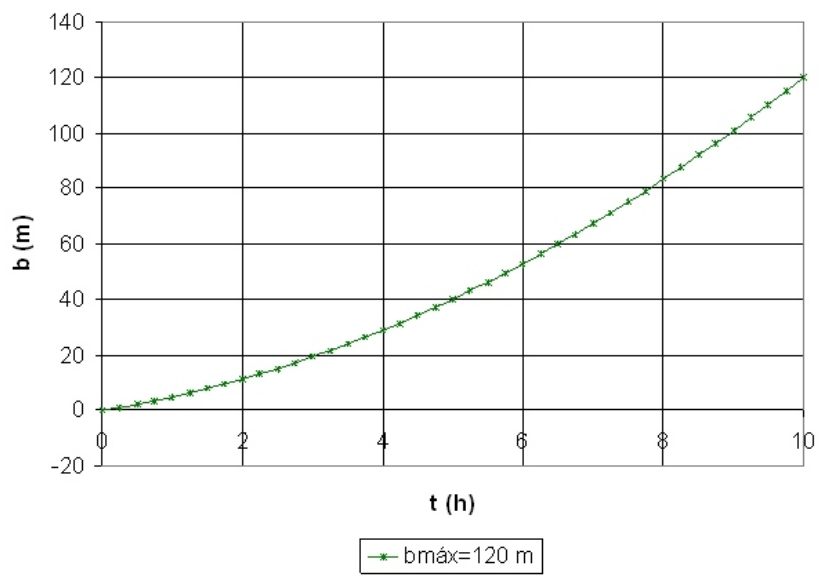

Figura 4. Ley de variación del ancho b en el tiempo $b=0.8 t^{2}+4 t-10^{-13}$

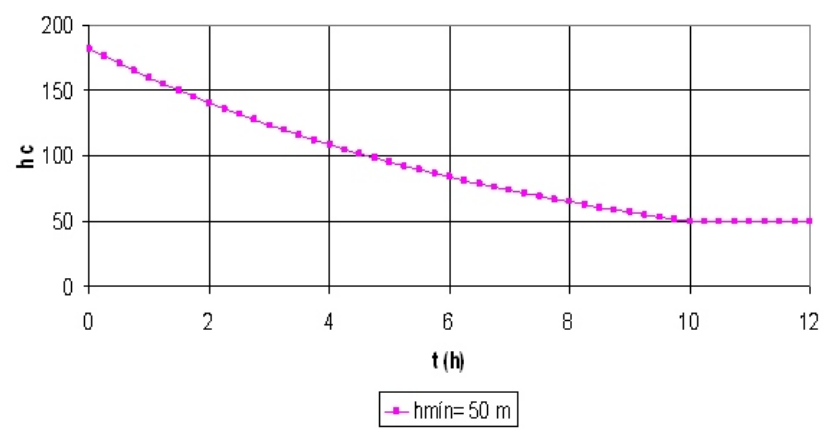

Figura 5. Ley de variación de la altura de la cortina en el tiempo $h_{c}=h_{c o r} e^{-0.7941 t}$ 


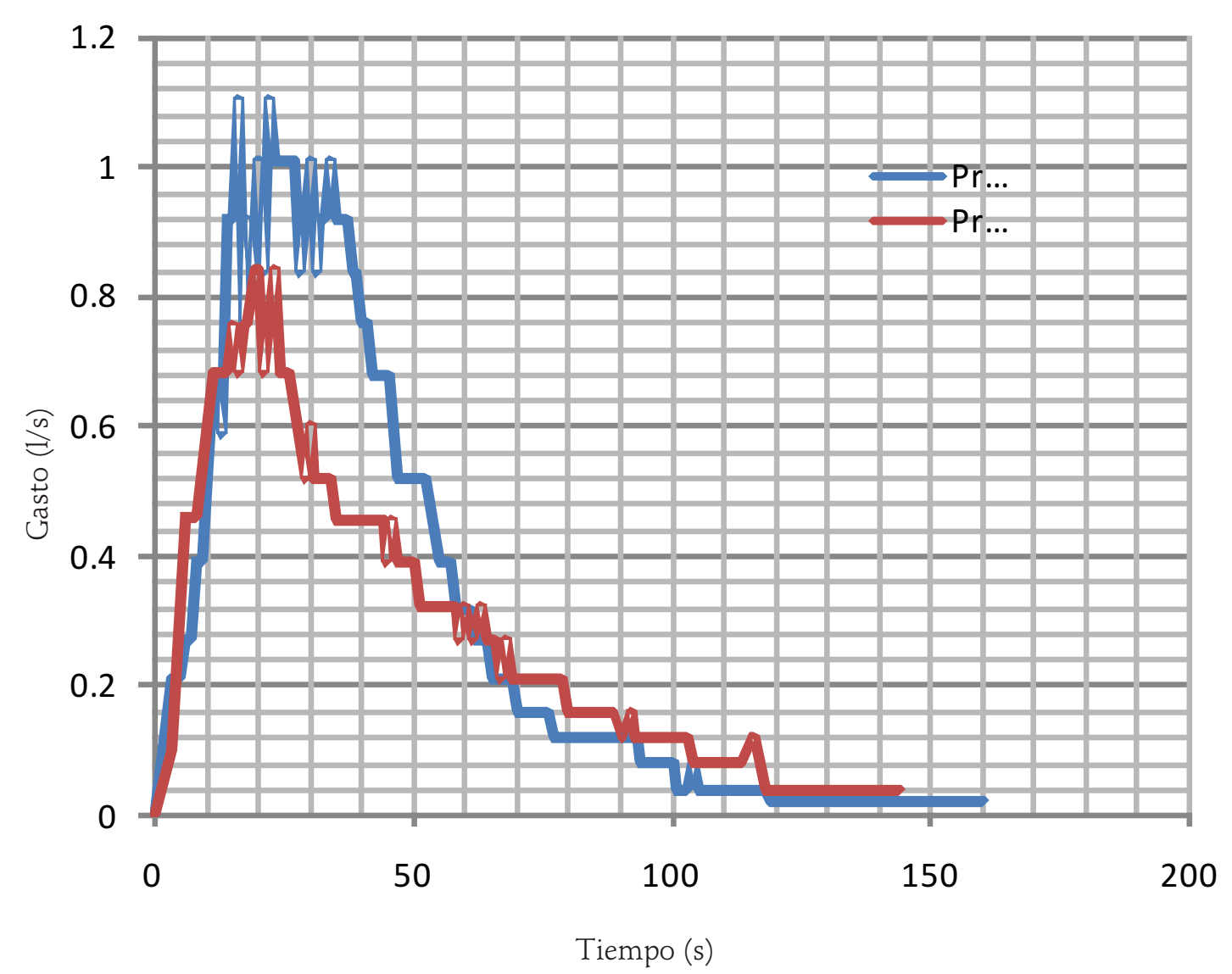

Figura 6. Ejemplo de hidrograma de salida por la ruptura de un bordo de arena volcánica, ensayado en laboratorio

\section{Aplicación}

Para calcular el hidrograma de salida durante la ruptura de la presa, se elaboró un programa en Visual Basic, que emplea la curva elevaciones-capacidades del embalse dada por la ecuación 1 y que la brecha de la rotura por donde sale del agua del embalse es de forma rectangular donde el ancho se modifica en el tiempo como se muestra en la figura 4 y que la altura de la brecha varía en el tiempo como se presenta en la figura 5 . En la figura 7 se muestra una carátula de arranque del programa de cómputo.
Ya que la sección máxima de la cortina de la presa la Parota vista en planta es de aproximadamente $806 \mathrm{~m}$ (figura 8), se propusieron otras formas de la variación en el tiempo del ancho de la brecha, así como de la altura de la cortina (figuras 9 y 10 ).

En la primera propuesta, se buscó que el volumen total almacenado en el embalse se desalojara en un tiempo aproximado de $10 \mathrm{~h}$; con un gasto de entrada al mismo de $30 \mathrm{~m}^{3} / \mathrm{s}$, en cualquier instante. 
DOI: http://dx.doi.org/10.22201/fi.25940732e.2010.11n3.023

Estimación numérica del hidrograma de salida de una presa debido a su ruptura

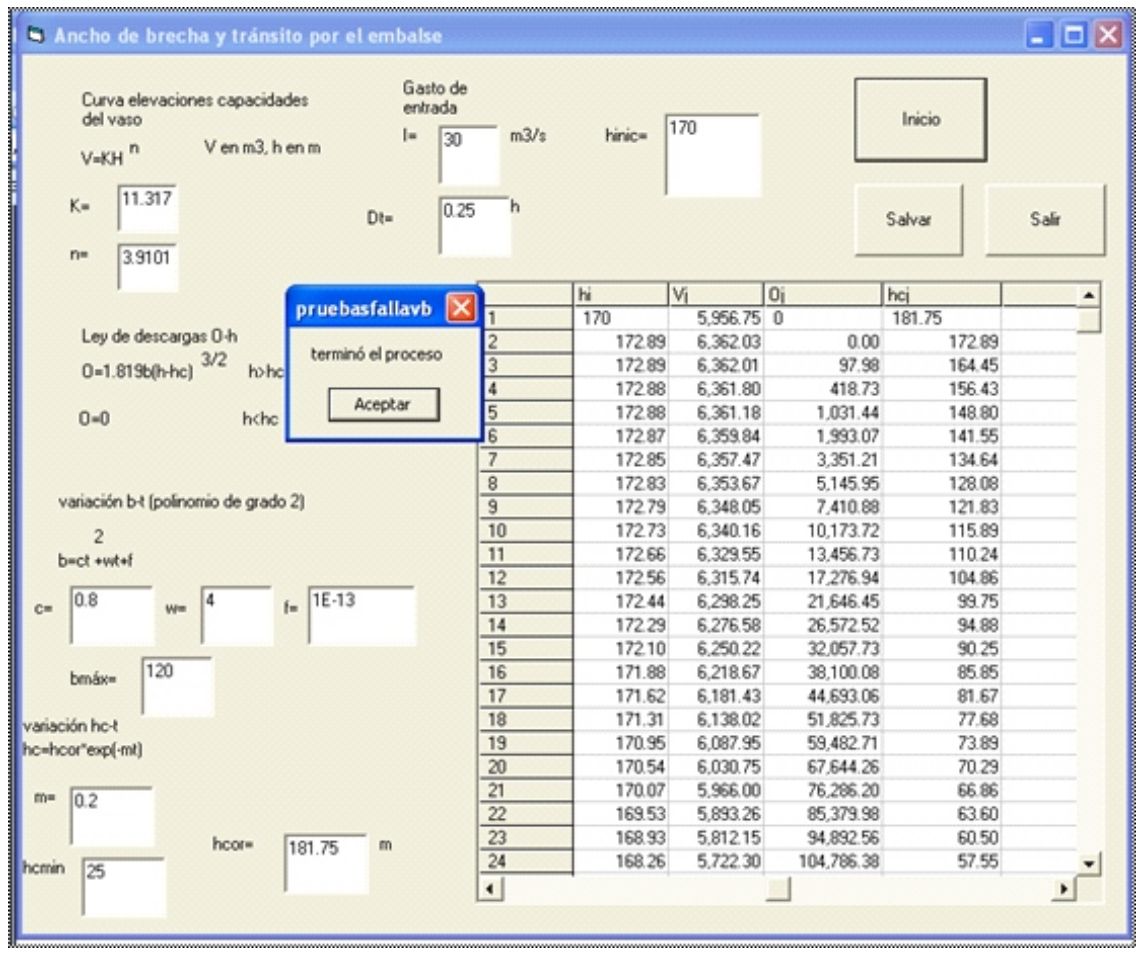

Figura 7. Carátula para proporciona $r$ datos y correr el Programa de tránsito de avenidas por el embalse ante una falla rectangular de una presa

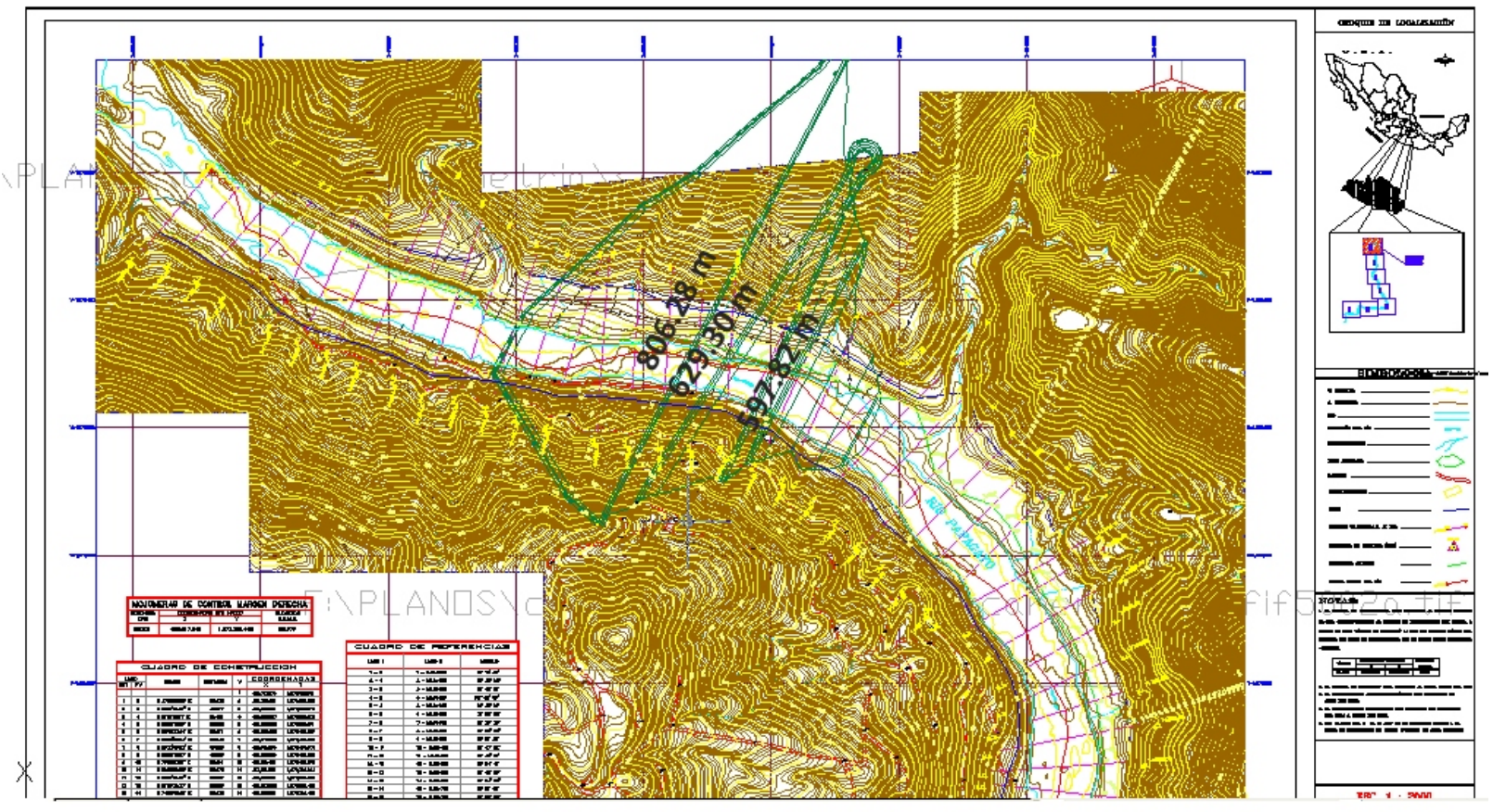

Figura 8. Vista en planta la cortina del P.H. La Parota, Guerrero 


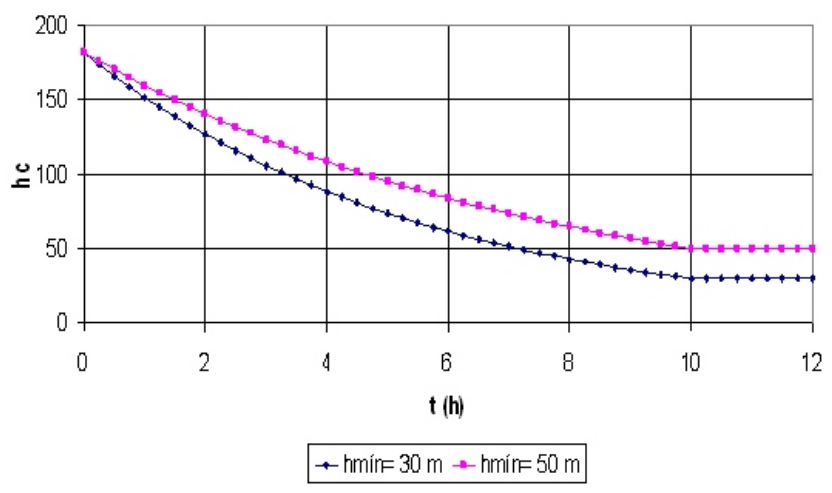

Figura 9. Variación de la altura de la cortina en el tiempo

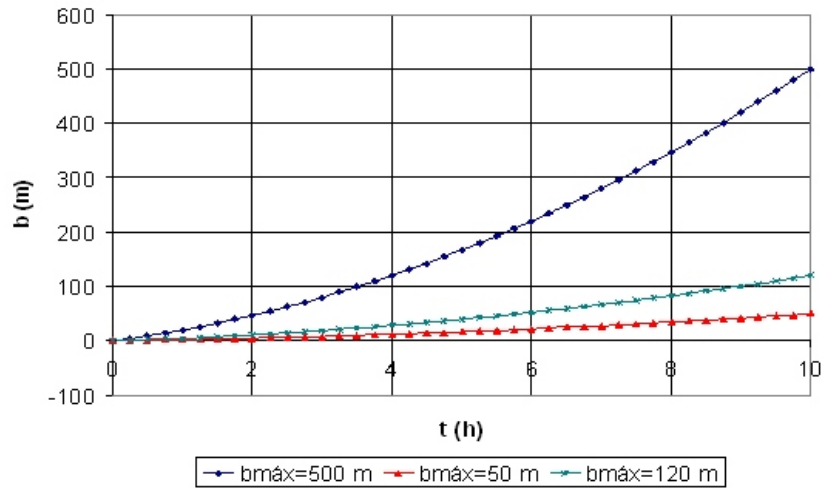

Figura 10. Variación del ancho de la falla en el tiempo

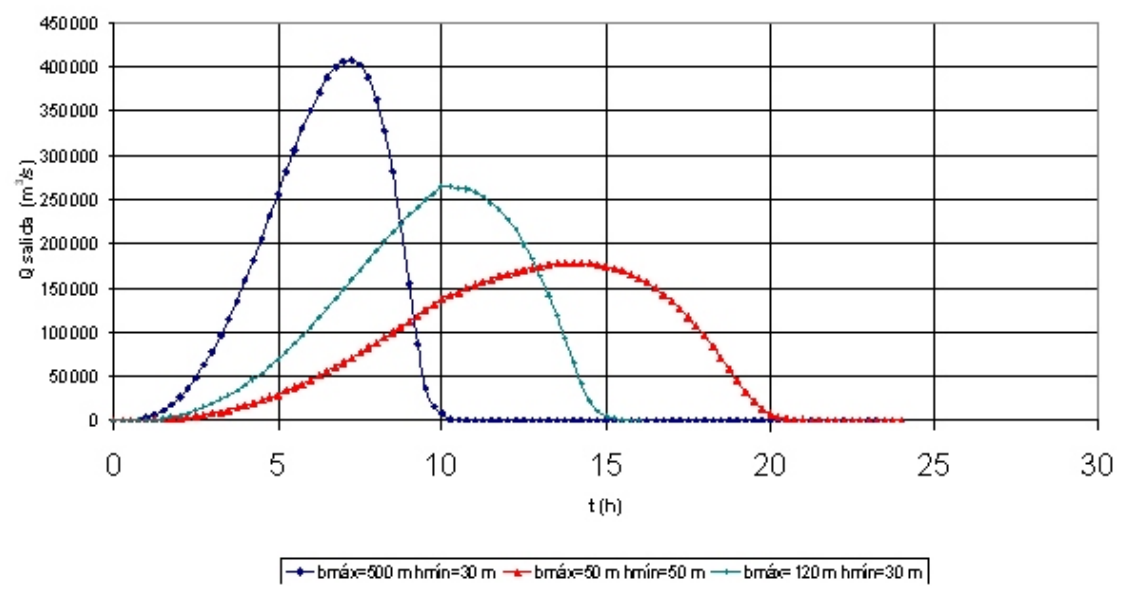

Figura 11. Hidrograma de salida debido a la ruptura de la presa

\section{Resultados y discusión}

Se obtuvieron tres hidrogramas de salida para los escenarios correspondientes a una situación desfavorable, una intermedia y un evento conservador que son mostrados en la figura 11.

En los hidrogramas se observa que el gasto y el tiempo del pico del hidrograma que sale por la falla de la cortina tienen un cambio importante. bla 1.

El resumen de estos resultados se consigna en la ta-

Los resultados contenidos en la tabla 1 muestran que el escenario más desfavorable podría descargarse a consecuencia de la eventual ruptura, de la cortina de la presa, un gasto de $407,853 \mathrm{~m}^{3} / \mathrm{s}$ que se presenta a las $7.25 \mathrm{~h}$ de iniciada la ruptura, siendo el caso más conservador; mientras que este gasto podría ser hasta de 178,408 m³ $/ \mathrm{s}$ en $14 \mathrm{~h}$ después de iniciada la ruptura (escenario optimista); por lo que podrían disponerse entre 7 y 14 horas para el alerta de los sitios aguas abajo de la cortina.

Tabla 1. Resumen de resultados considerando distintas leyes de variación en el ancho y en la altura de la falla

\begin{tabular}{|c|c|c|c|c|}
\hline \multicolumn{2}{|c|}{ Combinación } & \multirow{2}{*}{$\begin{array}{c}\mathrm{Qp} \\
\mathbf{m}^{3} / \mathbf{s}\end{array}$} & \multirow{2}{*}{$\begin{array}{c}\mathrm{Tp} \\
\mathrm{h}\end{array}$} & \multirow{2}{*}{$\begin{array}{c}\text { tb }^{\text {apróx }} \\
\mathbf{H}\end{array}$} \\
\hline bmáx m & hmín $m$ & & & \\
\hline 500 & 30 & 407853 & 7.25 & 10.25 \\
\hline 50 & 50 & 178409 & 14 & 20.5 \\
\hline 120 & 30 & 264615 & 10.25 & 15.5 \\
\hline
\end{tabular}

Posteriormente, los hidrogramas generados por la ruptura de la cortina de la presa fueron transitados por el cauce del río aguas abajo del embalse con un modelo numérico de alta resolución de simulación de flujo a superficie 
libre en cauces y que toma en cuenta los posibles cambios de régimen del flujo de subcrítico a supercrítico, el esquema numérico fue desarrollado en el Instituto de Ingeniería, UNAM (Fuentes et al., 2005). En las figuras 12 y 13 se presentan los perfiles hidráulicos a lo largo del cauce en tres tiempos distintos para dos casos estudiados, y en la tabla 2 los valores de algunas variables hidráulicas para el caso del hidrograma con gasto de pico de $178,409 \mathrm{~m}^{3} / \mathrm{s}$.

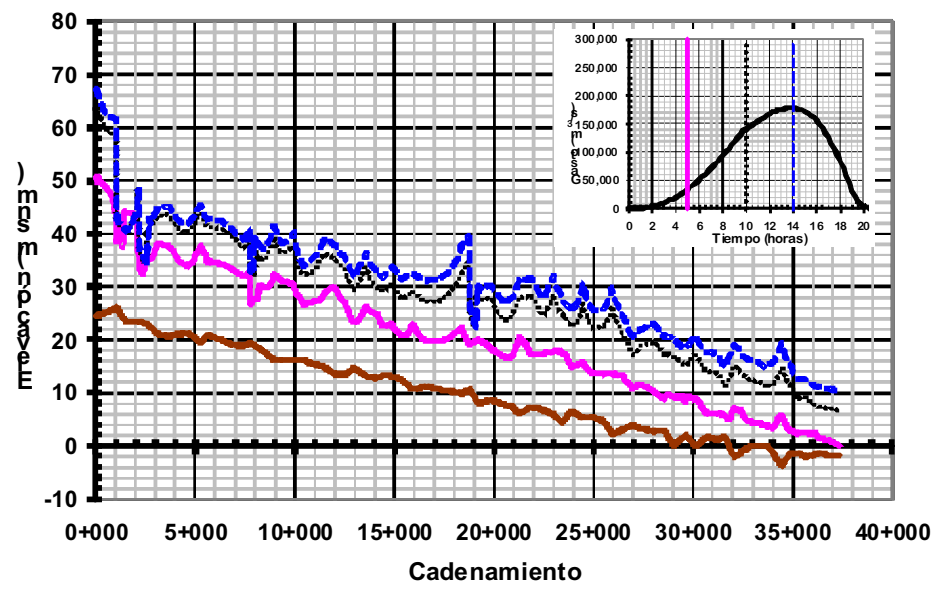

-TALWEG - SLA HORA 5 SLA HORA $10 \quad$-m SLAHORA 14

Figura 12. Funcionamiento hidráulico del río para el hidrograma con bmáx=50 $\mathrm{m}$ y hmín=50 $\mathrm{m}$

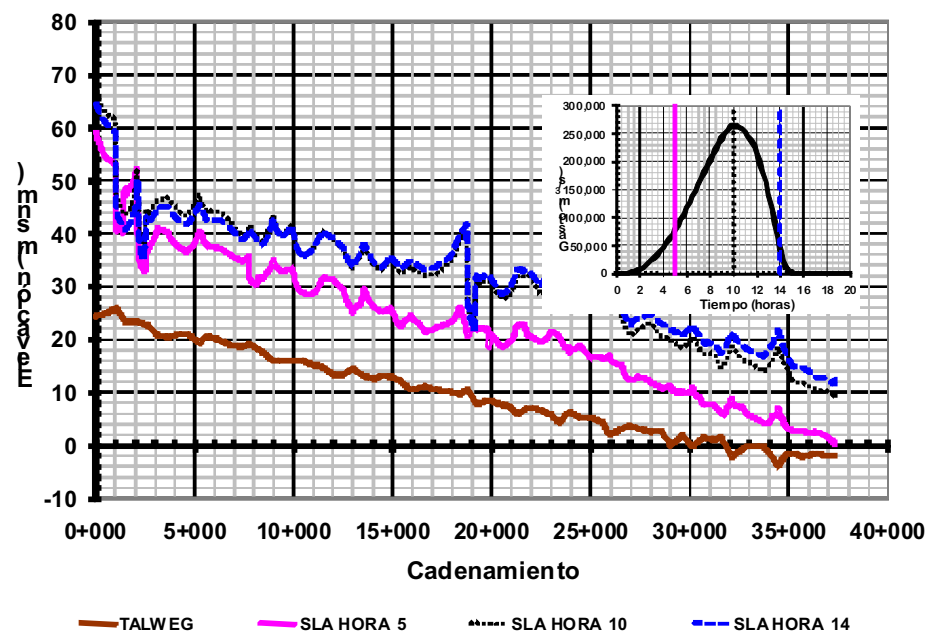

Figura 13. Funcionamiento hidráulico para el hidrograma con máx =120 m y hmin=30 m 
DOI: http://dx.doi.org/10.22201/fi.25940732e.2010.11n3.023

Fuentes-Mariles O.A., Arganis-Juárez M.L., De Luna-Cruz F. y Franco V.

Tabla 2. Resumen de resultados del tránsito de la avenida por el cauce

Tiempo: 5 horas. Hidrograma con bmáx $=50 \mathrm{~m}$ y hmin $=50 \mathrm{~m}$

\begin{tabular}{cccccccc}
\hline Sección & $\begin{array}{c}\text { Talweg+ } \\
\text { tirante } \\
(\mathrm{msnm})\end{array}$ & $\begin{array}{c}\text { Tirante } \\
(\mathrm{m})\end{array}$ & $\begin{array}{c}\text { Velocidad } \\
(\mathrm{m} / \mathrm{s})\end{array}$ & $\begin{array}{c}\text { Gasto } \\
\left(\mathrm{m}^{3} / \mathrm{s}\right)\end{array}$ & $\begin{array}{c}\text { Área } \\
\text { hidráulica } \\
\left(\mathrm{m}^{2}\right)\end{array}$ & $\begin{array}{c}\text { Radio } \\
\text { hidráulico } \\
(\mathrm{m})\end{array}$ & gAHT \\
\hline 1 & 50.73 & 26.23 & 8.15 & $33,864.47$ & $4,153.72$ & 17.28 & $445,464.13$ \\
2 & 49.83 & 25.28 & 7.13 & $34,755.60$ & $4,877.32$ & 15.37 & $435,646.66$ \\
3 & 50.54 & 25.94 & 5.09 & $33,410.54$ & $6,567.17$ & 14.60 & 504.763 .59 \\
4 & 50.75 & 26.10 & 3.91 & $31,533.40$ & $8,056.63$ & 14.00 & $551,599.19$ \\
5 & 50.01 & 25.31 & 4.27 & $33,106.54$ & $7,754.18$ & 14.15 & $522,574.91$ \\
6 & 49.89 & 25.14 & 3.89 & $32,234.16$ & $8,285.25$ & 14.30 & $535,073.88$ \\
7 & 49.65 & 24.85 & 3.86 & $31,972.92$ & $8,275.21$ & 14.44 & $530,007.63$ \\
8 & 49.41 & 24.56 & 4.00 & $32,071.69$ & $8,028.42$ & 14.59 & $519,607.66$ \\
9 & 49.23 & 24.33 & 4.10 & $31,767.80$ & $7,744.09$ & 14.74 & $510,323.31$ \\
10 & 49.07 & 24.12 & 4.32 & $31,703.57$ & $7,335.30$ & 14.88 & $498,728.53$ \\
11 & 48.91 & 23.91 & 4.38 & $31,117.98$ & $7,109.26$ & 15.03 & $492,599.31$ \\
12 & 48.60 & 23.51 & 4.30 & $31,159.12$ & $7,241.42$ & 14.85 & $486,400.34$ \\
13 & 48.46 & 23.29 & 4.10 & $30,625.25$ & $7,477.21$ & 14.68 & $487,459.16$ \\
\hline
\end{tabular}

\section{Conclusiones}

Se estimaron tres hidrogramas de salida de la presa La Parota que se presentarían durante una eventual ruptura de su cortina con un algoritmo numérico, basado en la ecuación de continuidad y apoyado con el método predictor corrector de Euler-Gauss, que permitió considerar distintas formas de evolución en el tiempo de la abertura de la cortina por donde sale el agua almacenada durante su fa1la. Se analizaron los resultados de tres escenarios para obtener el gasto máximo y tiempo base del hidrograma de egreso del embalse por la falla de la cortina. Se encontró que en los hidrogramas de salida, los gastos máximos descargados por la posible falla de la cortina gastos de pico estarían comprendidos entre los $178,409 \mathrm{~m}^{3} / \mathrm{s}$ y $407,853 \mathrm{~m}^{3} / \mathrm{s}$, con un tiempo base entre 7.25 y 14 horas. Los tránsitos de avenida por el cauce aguas abajo de la presa, mostraron que habría tirantes de agua de más de $30 \mathrm{~m}$ y que en algunos tramos del río se desbordaría el agua y se desplazaría hacia las zonas aledañas a él alcanzando a cubrir un área estrecha próxima al cauce con tirantes y velocidades pequeñas.

Los perfiles del flujo de agua mostraron gran variación en las elevaciones de la superficie libre a lo largo del cauce, debido a la gran irregularidad en las secciones transversales del río, lo que también está relacionado con cambios en el gasto y velocidades del flujo a lo largo del río. Se manejaron 781 de estas secciones del río que inicia en el sitio de proyecto y concluye en la desembocadura al mar.

El método numérico para simular el flujo en el cauce del río fue capaz de manejar el fuerte gradiente de la superficie libre con respecto a la longitud a lo largo del cauce, sobre todo cerca del embalse. Sin embargo, por el detalle de sus cálculos, para proporcionar una estimación adecuada de los volúmenes de agua que se desbordan, se requiere conocer el perfil topográfico de la corona de los bordos, tanto derecho como el izquierdo para precisar la elevación y anchos por donde sale el agua del río y realizar la determinación del valor de los gastos de desbordamiento de una manera más aproximada a su magnitud real. Desde luego, es fundamental disponer de secciones transversales más cercanas entre ellas y con mayor definición, ya que de ello dependen los tirantes y gastos del flujo de agua en el cauce.

\section{Referencias}

Aureli F., Mignosa P. Comparison Between Experimental and Numerical Results of 2D Flows Due to Levee-Breaking. On: 
DOI: http://dx.doi.org/10.22201/fi.25940732e.2010.11n3.023

Estimación numérica del hidrograma de salida de una presa debido a su ruptura

XXIX IAHR Congress Proceedings (Sept. 16-21, 2001, Beijing, China).

Chapra S.C. y Canale R.P. Métodos numéricos para ingenieros. Tercera edición. México. Mc Graw Hill. 1999.

Chinnarasri C., Jirakitlerd S., Wongwises S. Embankment Dam Breach and its Outflouw Characteristics. Civil Enginnering and Environmetnal Systems, 21(4):247-264. December 2004.

Coleman S.E., Andrews D.P., Wehhy M.G. Overtopping Breaching of Noncohesive Homogeneous Embankments. Journal of Hydraulic Engineering, ASCE, 128(9):829-838. 2002.

Dodson and Associates, Yung A.C. Inc. Technical Memorandum. Modeling a Dam Breach with HEC-1. USA. Dodson and Associates, Inc. 1997. Pp.9.
Fuentes M.O.A., Romo O.M.P., Sánchez S.F.J., Santoyo G.G.M, Domínguez M.R., Franco V., Gavilán E.J.J., Mayoral V.J.M., De Luna C.F., Arganis J.M.L., Palma N.A. y Cruz G.J.A. Modelo matemático de la ruptura de la cortina del P. H. La Parota y sus efectos aguas abajo. Informe final para Comisión Federal de Electricidad. 2005.

Wetmore J.N., Fread D.L., Wiele S.M. The NWS Simplified Dam Break Model Program Documentation, USA. [en línea] 1991. Disponible en: http://www.nws.noaa.gov/ohd/hrl/rvrmech/documenta tion/smpdbk. pdf\#search =' ${ }^{\prime}$ dam $\% 20$ break $\% 20$ modeling

Rivera D.C y Fuentes M.O.A. Pruebas de ruptura de un bordo homogéneo de arena volcánica. Informe interno. Instituto de Ingeniería, UNAM. México. Noviembre 2009.

\section{Semblanza de los autores}

Óscar A. Fuentes-Mariles. Egresado de la Facultad de Ingeniería de la Universidad Nacional Autónoma de México (UNAM), con maestría y doctorado por la misma institución. Actualmente es investigador de la Coordinación de Hidráulica del Instituto de Ingeniería en donde ha colaborado desde 1973. Asimismo, desde ese mismo año ha sido profesor de asignatura en la Facultad y en el Posgrado de Ingeniería de la UNAM. Se destaca por sus aportaciones a los métodos numéricos aplicados a la hidráulica, hidráulica marítima, hidráulica fluvial, además de su participación en el Centro Nacional de Prevención de Desastres.

Maritza L. Arganis-Juárez. Egresada con maestría y doctorado por la Facultad de Ingeniería, UNAM. En 1993, inició su colaboración con la UNAM como profesora de física en la Escuela Nacional Preparatoria. Actualmente es investigadora de la Coordinación de Hidráulica del Instituto de Ingeniería de la UNAM, en la que realiza investigaciones en el área de aprovechamientos hidráulicos e hidrología. También es profesora de asignatura definitiva de las asignaturas Análisis Numérico y Cinemática, así como Dinámica en la Facultad de Ingeniería de la UNAM.

Faustino de Luna-Cruz. Egresado de la Escuela Nacional de Estudios Profesionales "Acatlán" de la UNAM, realizó la maestría en ingeniería hidráulica por la División de Estudios de Posgrado de la Facultad de Ingeniería, UNAM. Desde 1996, colabora en la Coordinación de Hidráulica desarrollándose en el área de hidráulica urbana y de análisis de flujos en cauces, así como proyectos de análisis de obras de protección contra inundaciones. Actualmente es técnico académico del Instituto de Ingeniería, UNAM.

Victor Franco. Es egresado de la Facultad de Ingeniería, UNAM con maestría por la misma institución. Actualmente es investigador de la Coordinación de Hidráulica del Instituto de Ingeniería, profesor de asignatura de la Facultad de Ingeniería y del Posgrado en Ingeniería de la UNAM desde 1975. Sus áreas de investigación son en materia de hidráulica fluvial y obras hidráulicas. Ha participado en varios modelos físicos de obras de excedencias de algunas de las grandes presas de México. 